\title{
SOME THEOREMS IN PROBABILITY THEORY
}

\author{
Bernard R. Gelbaum
}

In memory of Ernst Straus

\begin{abstract}
The following miscellany deals with questions of stochastic independence, normality, and "quantum mechanical" probability. The author was a close and admiring friend of Professor Straus from the time in 1941, when he first came to the United States (on the last ship from Palestine), until his untimely and tragic death in 1983.
\end{abstract}

0. Introduction. In the modern definitions $[6,7]$ of Gaussian processes the model is that of a real Hilbert space $\mathscr{H}$, a (probability) measure situation $(S, \mathscr{S}, P)$ and a linear isometry $T: \mathscr{H} \mapsto L^{2}(S)$ such that for all $f$ in $\mathscr{H}, \int_{S}(T f)(x) d P=0$, such that orthogonal elements $f, g$ in $\mathscr{H}$ map into (stochastically) independent random variables $T(f), T(g)$, and such that each nonzero $T f$ is normally distributed, i.e.,

$$
P\{x:(T(f))(x) \leq t\}=\frac{1}{\sqrt{2 \pi}\|T f\|_{2}} \int_{-\infty}^{t} \exp \left(-y^{2} / 2\|T f\|_{2}^{2}\right) d y .
$$

Among the consequences of the results that follow is the fact that the last two requirements stated above are mutually redundant (see §3).

The underlying phenomenon is found in the following:

THEOREM 1. If $X, Y \in L^{2}(S)$, if $X$ and $Y$ are independent and nonconstant and if $X \pm Y$ are independent then $X$ and $Y$ are normally distributed (and hence so are $X \pm Y$ ).

The works of Skitovich and Darmois $[1,8,9]$ imply this theorem but it and some of its natural generalizations are derived below by methods somewhat different from those used in $[1,8,9]$. Along the way there are proved some analytical lemmas of independent interest.

1. The proof of Theorem 1. Since $X, Y \in L^{2}(S)$ it follows that $X, Y \in L^{1}(S)$ and thus they may be normalized according to the transformation:

$$
X \mapsto\left(X-\int_{S} X(x) d P\right) /\left\|X-\int_{S} X(x) d P\right\|_{2}=\tilde{X}
$$


Then $\tilde{X}, \tilde{Y}$ are independent and nonconstant and the hypothesis $r e X \pm Y$ implies $\tilde{X} / \sqrt{2} \pm \tilde{Y} / \sqrt{2}$ are independent. If $\varphi$ and $\psi$ are the respective characteristic functions of $\tilde{X}$ and $\tilde{Y}$, e.g., $\varphi(t)=\int_{S} e^{i t \tilde{X}} d P$, and if

$$
U=\frac{\tilde{X}}{\sqrt{2}}+\frac{\tilde{Y}}{\sqrt{2}}, \quad V=\frac{\tilde{X}}{\sqrt{2}}-\frac{\tilde{Y}}{\sqrt{2}},
$$

then $U$ and $V$ are independent,

$$
\tilde{X}=\frac{U}{\sqrt{2}}+\frac{V}{\sqrt{2}}, \quad \tilde{Y}=\frac{U}{\sqrt{2}}-\frac{V}{\sqrt{2}},
$$

and if $\xi$ and $\mu$ are the respective characteristic functions of $U$ and $V$, the conditions of independence imply:

$$
\begin{aligned}
& \xi(t)=\varphi(t / \sqrt{2}) \psi(t / \sqrt{2}) \\
& \mu(t)=\varphi(t / \sqrt{2}) \overline{\psi(t / \sqrt{2})} \\
& \varphi(t)=\xi(t / \sqrt{2}) \mu(t / \sqrt{2}) \\
& \psi(t)=\xi(t / \sqrt{2}) \overline{\mu(t / \sqrt{2})}
\end{aligned}
$$

Replacing $\xi$ and $\mu$ in (2) by their formulae given by (1) yields:

$$
\begin{aligned}
& \varphi(t)=\varphi\left(t /(\sqrt{2})^{2}\right) \psi\left(t /(\sqrt{2})^{2}\right) \varphi\left(t /(\sqrt{2})^{2}\right) \overline{\psi\left(t /(\sqrt{2})^{2}\right)} \\
& \psi(t)=\varphi\left(t /(\sqrt{2})^{2}\right) \psi\left(t /(\sqrt{2})^{2}\right) \overline{\varphi\left(t /(\sqrt{2})^{2}\right)} \psi\left(t /(\sqrt{2})^{2}\right)
\end{aligned}
$$

or

$$
\begin{aligned}
& \varphi(t)=\left[\varphi\left(t /(\sqrt{2})^{2}\right)\right]^{2}\left|\psi\left(t /(\sqrt{2})^{2}\right)\right|^{2} \\
& \psi(t)=\left|\varphi\left(t /(\sqrt{2})^{2}\right)\right|^{2}\left[\psi\left(t /(\sqrt{2})^{2}\right)\right]^{2} .
\end{aligned}
$$

Mathematical induction applied to the process of replacing $\varphi, \psi$ in the right members in (3) by the formulae provided by (3) yields the following general formula:

$$
\begin{aligned}
\varphi(t)= & {\left[\varphi\left(t /(\sqrt{2})^{2^{n}}\right) /\left|\varphi\left(t /(\sqrt{2})^{2^{n}}\right)\right|\right]^{2^{2 n-1}} } \\
& \cdot\left|\varphi\left(t /(\sqrt{2})^{2^{n}}\right)\right|^{2^{2^{n}-1}} \cdot\left|\psi\left(t /(\sqrt{2})^{2^{n}}\right)\right|^{2^{2^{n}-1}}, \quad n=1,2, \ldots,
\end{aligned}
$$

and a similar formula for $\psi$.

Since

$$
\varphi(t), \psi(t)=\left(1-\frac{\|\tilde{X}\|_{2}^{2} t^{2}}{2}+o\left(t^{2}\right)\right)
$$


it follows, since also $\|\tilde{X}\|_{2}=1$, that $\varphi(t)=e^{-t^{2} / 2}=\psi(t)$. And so the normality of $X$ (and similarly of $Y$ ) is proved.

2. Generalization. Although Theorem 1 appears to be quite special, the line of its proof may be imitated in a far more general setting. If $X, Y$ are in $L^{2}(S)$ and independent and if $U=a X+b Y, V=c X+d Y$ are also independent then $X$ and $Y$ may be normalized (so that $\int_{S} X d P=$ $\int_{S} Y d P=0$ and $\|X\|_{2}=\|Y\|_{2}=1$ ) without disturbing the conclusion. Then $\int_{S} U d P=\int_{S} V d P=0$ and so after multiplication by suitable constants the original hypothesis may be rephrased: $X, Y$ are independent, $U$, $V$ are independent and $a^{2}+b^{2}=c^{2}+d^{2}=1, a c+b d=0$. As given in the preceding sentence the hypothesis does not imply that $X$ or $Y$ or $U$ or $V$ is normal (e.g., $a=d=1, b=c=0, X, Y$ arbitrary and independent). However, if the hypothesis, which already states that $\left(\begin{array}{ll}a & b \\ c & d\end{array}\right)$ is an orthogonal matrix, also requires that it represents a rotation through an angle $\theta$ that is not an integral multiple of $\pi / 2$, then the conclusion that $X, Y, U, V$ are all normal is forced. The supplementary requirement avoids triviality and without loss of generality is simply a normalization.

THEOREM 2. If $X, Y$ are in $L^{2}(S)$ and independent, $\int_{S} X d P=\int_{S} Y d P$ $=0,\|X\|_{2}=\|Y\|_{2}=1$, if $\theta$ is such that $\cos \theta \cdot \sin \theta \neq 0$, and if $U=$ $X \cos \theta+Y \sin \theta, V=-X \sin \theta+Y \cos \theta$ are independent, then $U, V, X$, and $Y$ are all normally distributed.

Proof. As in the proof of Theorem 1, it is readily established that (with the notations, $c, s$ for $\cos \theta, \sin \theta$ )

$$
\begin{aligned}
\xi(t) & =\varphi(c t) \psi(s t) \\
\mu(t) & =\overline{\varphi(s t)} \psi(c t) \\
\varphi(t) & =\xi(c t) \overline{\mu(s t)} \\
\psi(t) & =\xi(s t) \mu(c t) .
\end{aligned}
$$

Then the substitution procedure leads to

$$
\begin{aligned}
& \varphi(t)=\varphi\left(c^{2} t\right) \psi(c s t) \cdot \varphi\left(s^{2} t\right) \overline{\psi(c s t)}=\varphi\left(c^{2} t\right)|\psi(c s t)|^{2} \varphi\left(s^{2} t\right) \\
& \psi(t)=\varphi(c s t) \psi\left(s^{2} t\right) \cdot \overline{\varphi(c s t)} \psi\left(c^{2} t\right)=\psi\left(c^{2} t\right)|\varphi(c s t)|^{2} \psi\left(s^{2} t\right) .
\end{aligned}
$$

This time, the repeated process of replacement does not yield the simple formulas found earlier. But the fact that $\varphi(t), \psi(t)=\left(1-t^{2} / 2+o\left(t^{2}\right)\right)$ 
allows the conclusion (established by induction)

$$
\begin{gathered}
\varphi(t)=\prod_{k=0}^{n}\left(1-\left(\begin{array}{l}
n \\
k
\end{array}\right)\left(c^{2}\right)^{n-k}\left(s^{2}\right)^{k} \frac{t^{2}}{2}\right)+E_{n}\left(t^{2}\right), \quad n=1,2, \ldots, \\
E_{n}\left(t^{2}\right) \rightarrow 0 \quad \text { as } n \rightarrow \infty .
\end{gathered}
$$

A similar formula obtains for $\psi$.

At this point the following general lemmas are useful.

LEMMA 1. If $\left(t_{n k}\right)$ is a Toeplitz matrix $\left(\sum_{k=1}^{\infty} t_{n k} \rightarrow 1\right.$ as $n \rightarrow \infty$, $t_{n k} \rightarrow 0$ as $\left.n \rightarrow \infty, \sum_{k=1}^{\infty}\left|t_{n k}\right| \leq M<\infty\right)$ and if furthermore $\sum_{k=1}^{\infty} t_{n k}^{2} \rightarrow 0$ as $n \rightarrow \infty$ then for all complex $z, \prod_{k=1}^{\infty}\left(1+t_{n k} z\right) \rightarrow e^{z}$ as $n \rightarrow \infty$.

Remarks. The condition that $\sum_{k=1}^{\infty} t_{n k}^{2} \rightarrow 0$ as $n \rightarrow \infty$ cannot be dropped, e.g., if $t_{n k}=\delta_{n k}$ the conclusion is false. The special case $t_{n k}=$ $1 / n, k=1,2, \ldots, n, t_{n k}=0, k>n$, should be noted as related to the formula $e^{z}=\lim _{n \rightarrow \infty}(1+z / n)^{n}$.

Proof. Since $\sum_{k=1}^{\infty}\left|t_{n k}\right| \leq M<\infty$, the infinite product $\prod_{k=1}^{\infty}\left(1+t_{n k} z\right)$ converges for all $z$ in $\mathbf{C}$ and represents an entire function $f_{n}(z)$. Furthermore, if $0<\delta<1 / M$ there are functions $\alpha_{n}(z)$ such that for all $n$ and all $z$ such that $|z| \leq \delta,\left|\alpha_{n}(z)\right| \leq K<\infty$ and

$$
\exp \left(t_{n k} z-\left(t_{n k}^{2} / 2\right) z^{2} \alpha_{n}(z)\right)=\left(1+t_{n k} z\right) .
$$

Hence if $|z| \leq \delta$ and $s_{n}=\sum_{k=1}^{\infty} t_{n k}^{2}$ then

$$
f_{n}(z)=\prod_{k=1}^{\infty}\left(1+t_{n k} z\right)=\exp \left(\sum_{k=1}^{\infty} t_{n k} z-\frac{s_{n} \alpha_{n}(z)}{2}\right) \rightarrow e^{z} \text { as } n \rightarrow \infty .
$$

Since the $f_{n}(z)$ and $e^{z}$ are entire, $f_{n}(z) \rightarrow e^{z}$ as $n \rightarrow \infty$ for all $z$.

LEMMA 2. If $0<a, b, a+b=1$, and if $t_{n k}=\left(\begin{array}{l}n \\ k\end{array}\right) a^{n-k} b^{k}$ then $\left(t_{n k}\right)$ is a Toeplitz matrix such that $\sum_{k=1}^{\infty} t_{n k}^{2} \rightarrow 0$ as $n \rightarrow \infty$.

Proof. That $\left(t_{n k}\right)$ is a Toeplitz matrix is immediate. The proof that $\sum_{k=1}^{\infty} t_{n k}^{2} \rightarrow 0$ as $n \rightarrow \infty$ is divided into two cases.

Case 1. $a=b=\frac{1}{2}$. Then $t_{n k}^{2}=\left(\begin{array}{c}n \\ k\end{array}\right)^{2} 2^{-2 n}$. If $n$ is even, $n=2 m$ then $\max _{k}\left(\begin{array}{l}n \\ k\end{array}\right) 2^{-2 n}$ is by Stirling's formula

$$
\left(\begin{array}{c}
2 m \\
m
\end{array}\right) 2^{-4 m} \doteq\left(1+\delta_{m}\right) \frac{2^{-2 m}}{\sqrt{\pi m}}, \quad \delta_{m} \rightarrow 0 \text { as } m \rightarrow \infty .
$$


Thus since $\sum_{k=1}^{\infty}\left(\begin{array}{l}n \\ k\end{array}\right) / 2^{n}=1$ and $\left(\begin{array}{l}n \\ k\end{array}\right) / 2^{n}>0$, it follows that

$$
\sum_{k=0}^{n} \frac{\left(\begin{array}{l}
n \\
k
\end{array}\right)^{2} 2^{-2 n}}{2^{n}} \leq \frac{2^{-2 m}}{\sqrt{\pi m}}\left(1+\delta_{m}\right)
$$

and so

$$
0<\sum_{k=0}^{n}\left(\begin{array}{l}
n \\
k
\end{array}\right)^{2} 2^{-2 n} \leq \frac{1+\delta_{m}}{\sqrt{\pi m}} \rightarrow 0 \quad \text { as } m \rightarrow \infty .
$$

If $n$ is odd a similar calculation yields the same conclusion.

Case 2. $0<a<b, c=b / a>1$. Then

$$
\sum_{k=0}^{n}\left(\begin{array}{l}
n \\
k
\end{array}\right)^{2} a^{2 n-2 k} b^{2 k}=a^{2 n} \sum_{k=0}^{n}\left(\begin{array}{l}
n \\
k
\end{array}\right)^{2} c^{2 k} \text {. }
$$

The last term of the sum is $c^{2 n}$, the first term of the sum is 1 and if $0<k<n$ the ratio

$$
c^{2 n} /\left(\begin{array}{l}
n \\
k
\end{array}\right)^{2} c^{2 k}
$$

is, again by Stirling's formula

$$
\left(1+\mu_{k n}\right) k^{2 k+1}\left(1-\frac{k}{n}\right)^{2 n} \cdot 2 \pi \cdot(1-k / n) \cdot \frac{1}{(n-k)^{2 k}} c^{2 n-2 k}
$$

$\left(\mu_{k n} \rightarrow 0\right.$ as $\left.n \rightarrow \infty\right)$ which is unbounded as $n \rightarrow \infty$. Hence for large $n$, $c^{2 n}$ is the largest term of the sum and so the sum is not more than $(n+1) c^{2 n}$. Consequently, since $0<b<1$,

$$
0 \leq a^{2 n} \sum_{k=0}^{n}\left(\begin{array}{l}
n \\
k
\end{array}\right)^{2} c^{2 k} \leq(a c)^{2 n}(n+1)=b^{2 n}(n+1) \rightarrow 0
$$

as $n \rightarrow \infty$.

In the proof of the theorem itself the lemmas above show that (with $a=c^{2}, b=s^{2}$ )

$$
\varphi(t)=e^{-t^{2} / 2}
$$

as required.

3. Gaussian processes. In [6, 7] a Gaussian process is defined as a (linear) isometry $T: \mathscr{H} \rightarrow L_{\mathbf{R}}^{2}(S)$ of a (real) Hilbert space $\mathscr{H}$ into the (equivalence) classes of square-integrable $\mathbf{R}$-valued functions on a probability sample space $S$. Furthermore, it is required that if $x, y$ in $\mathscr{H}$ are 
orthogonal then $T(x), T(y)$ are independent and that each $T(x)$ is normally distributed with mean 0 and variance $(\operatorname{var}(T(x)))\|x\|^{2}$. Theorem 1 shows that the requirement that each $T(x)$ be normally distributed is redundant.

Indeed, if $x$ and $y$ are orthogonal so are $x \pm y$ whence $T(x)=X$ and $T(y)=Y$ are independent as are $X \pm Y$ and Theorem 1 implies they are normally distributed. The fact that $T$ is an isometry implies that $\operatorname{var}(T(x))$ $=\|x\|^{2}$. On the other hand, the requirement $\operatorname{var}(T(x))=\|x\|^{2}$ implies that $T$ is an isometry and the additional requirement that $T(x)$ be distributed normally implies that orthogonal $x, y$ go into orthogonal $T(x)$, $T(y)$, i.e., orthogonal normally distributed random variables having covariance 0 , hence independent.

The (finitely additive) Gaussian cylinder measure induced on $\mathscr{H}$ by $T$ is not extendable to a countably additive measure on the $\sigma$-algebra $Z_{\mathscr{H}}$ generated by the cylinders of $\mathscr{H}$ if $\mathscr{H}$ is infinite-dimensional. On the other hand, if $S$ is a domain in $\mathbf{C}$, if the two-dimensional Lebesgue measure of $S$ is finite (and normalized so that it is 1 ), if $\mathscr{H}$ is the set of $\mathbf{R}$-valued square-integrable harmonic functions on $S$, and if $T$ is any bounded endomorphism of $\mathscr{H}$, then [4] $T$ induces a countably additive measure on $Z_{\mathscr{H}}$. Thus no such $T: \mathscr{H} \rightarrow \mathscr{H}$ can satisfy either of the following requirements:

(a) if $(x, y)=0$ then $T(x), T(y)$ are independent;

(b) each $T(x)$ is normally distributed and $\operatorname{var}(T(x))=k\|x\|^{2}(k$ independent of $x)$.

It should be noted also that if $\left\{X_{\lambda}\right\} \subset L^{2}(S)$ ( $S$ arbitrary), if $L^{2}(S)$ is infinite-dimensional, and if all (finite-dimensional) joint distribution functions for the $X_{\lambda}$ are (multidimensional) normal, then $\left[\operatorname{span}\left\{X_{\lambda}\right\}\right]^{\perp}$ is infinite-dimensional. The proof follows.

If $\left\{Y_{n}\right\}_{n=1}^{N}$ is an orthonormal subset of $\overline{\operatorname{span}\left\{X_{\lambda}\right\}}$ then each $Y_{n}$ is a limit of finite linear combinations of the $X_{\lambda}$ and the joint distribution function of the $Y_{n}$ is also multidimensional normal while all covariances are 0 . Thus the $Y_{n}$ are independent. Since there is an orthonormal subset $\left\{Y_{\mu}\right\}$ of $\overline{\operatorname{span}\left\{X_{\lambda}\right\}}$ and for which $\overline{\operatorname{span}\left\{Y_{\mu}\right\}}=\overline{\operatorname{span}\left\{X_{\lambda}\right\}}$ it follows [2] that $\left[\operatorname{span}\left\{X_{\lambda}\right\}\right]^{\perp}$ is infinite-dimensional.

The last result gives rise to the following question:

1. Are there useful ways of stating conditions on the set of finite-dimensional joint distribution functions $\left\{F_{\lambda_{1}, \ldots, \lambda_{n}}\left(x_{\lambda_{1}}, \ldots, x_{\lambda_{n}}\right)\right\}$ for the set $\left\{X_{\lambda}\right\}$ so that these conditions are necessary and/or sufficient for the truth of $\left[\operatorname{span}\left\{X_{\lambda}\right\}\right]^{\perp}=\{0\}$ ? 
4. Quantum mechanical probability. Associated with each observable $\mathscr{R}$ in quantum theory is a resolution of the identity $E(t),-\infty<t<\infty$, and with each state $f$ (a unit vector in Hilbert space $\mathscr{H}$ ) there is associated a distribution function $F_{f}$ defined by: $F_{f}(t)=(E(t) f, f),[10]$. The number $F_{f}(t)$ is interpreted as the probability that in the state $f$ the observable $\mathscr{R}$ assumes a value in $(-\infty, t]$. As a monotone increasing function $F_{f}$ is differentiable almost everywhere. If $S_{f}=\left\{t_{0}: F_{f}^{\prime}\left(t_{0}\right)\right.$ exists $\}$ then the Lebesgue measure $m\left(\mathbf{R} \backslash S_{f}\right)=0$. On the other hand if $\operatorname{supp}(E(t))=$ $\mathbf{R} \backslash\{(a, b): a<b, E(b)=E(a)\}$, then there obtains:

THEOREM 2. Let $K$ be a set of the second category in $\Sigma_{1}$, the surface of the unit spherre of $\mathscr{H}$, or in $\mathscr{H}$ itself. Then

$$
\operatorname{supp}(E(t)) \cap\left(\bigcap_{f \text { in } K} S_{f}\right)=\varnothing .
$$

Interpretation: For each $f$ in $\Sigma_{1}$, for each positive $e$, and for each $t_{0}$ in $S_{f}$ there is in $\Sigma_{1}$ a $g$ such that $\|f-g\|<e$ and $F_{g}$ is not differentiable at $t_{0}$. Thus $F_{f}$ is and $F_{g}$ is not differentiable at $t_{0}$ even though $f$ and $g$ are close.

Proof. For $t_{0}$ in $\operatorname{supp}(E(t))$ if $F_{f}^{\prime}\left(t_{0}\right)$ exists for all $f$ in $K$ then

$$
\lim _{h \rightarrow 0}\left(\left(E\left(t_{0}+h\right)-E\left(t_{0}\right)\right) f, f\right) / h
$$

exists in $K$. The Banach-Steinhaus theorem implies there is an $\mathbf{R}$ an $M$ such that

$$
\left\|E\left(t_{0}+h\right)-E\left(t_{0}\right)\right\| / h \leq M
$$

for all $h$ near 0 . Since $E\left(t_{0}+h\right)-E\left(t_{0}\right)$ is idempotent it follows that

$$
\left\|E\left(t_{0}+h\right)-E\left(t_{0}\right)\right\| / h \leq h M^{2}
$$

whence

$$
\lim _{h \rightarrow 0}\left\|E\left(t_{0}+h\right)-E\left(t_{0}\right)\right\| / h=0 .
$$

However if $t_{0}$ is in $\operatorname{supp}(E(t))$ then either (a) $t_{0}$ is an isolated point of the set or (b) there is a sequence $\left\{t_{n}\right\}_{n=1}^{\infty}$ such that $t_{n} \uparrow t_{0}$ (or $t_{n} \downarrow t_{0}$ ) and $\left(E\left(t_{n+1}\right)-E\left(t_{n}\right)\right) \neq 0$. If (a) is true then there is an $\Sigma_{1}$ an $f$ such that $E\left(t_{0}\right) f=f$ and if $t<t_{0}$, then $E(t) f=0$. Hence if $h$ is small and positive

$$
\left(\left(E\left(t_{0}-h\right)-E\left(t_{0}\right)\right) f, f\right) / h=-1 / h
$$

and so

$$
\left\|E\left(t_{0}-h\right)-E\left(t_{0}\right)\right\| / h \nrightarrow 0 \text { as } h \rightarrow 0
$$


If (b) is true, for each $n$ in $\mathbf{N}$ there is in $\Sigma_{1}$ an $f_{n}$ such that $\left(E\left(t_{n+1}\right)-E\left(t_{n}\right)\right) f_{n}=f_{n}$ and $\left\{f_{n}\right\}_{n=1}^{\infty}$ is orthonormal. If

$$
b_{n}=\left(\left(t_{n}-t_{n-1}\right) /\left(t_{1}-t_{0}\right)\right)^{1 / 2}
$$

$n$ in $\mathbf{N}$, then $\sum_{n=1}^{\infty} b_{n}^{2}=1$ and $\sum_{n=1}^{\infty} b_{n} f_{n}=f$ is in $\Sigma_{1}$. On the other hand

$$
\sum_{n=m}^{\infty}\left(\left(E\left(t_{n}\right)-E\left(t_{n+1}\right)\right) f, f\right)=-\frac{\left(t_{m}-t_{0}\right)}{\left(t_{1}-t_{0}\right)} .
$$

The left member above is also $\left(\left(E\left(t_{m}\right)-E\left(t_{0}\right)\right) f, f\right)$ and so if $F_{f}^{\prime}\left(t_{0}\right)$ exists it must be $-\left(t_{1}-t_{0}\right)^{-1}$ which is not 0 , and a contradiction emerges. The result follows.

THEOREM 3. If $\mathscr{H}$ is separable there is an $\mathscr{H}$ a sequence $\left\{f_{n}\right\}_{n=1}^{\infty}$ dense in $\mathscr{H}\left(\right.$ or in $\left.\Sigma_{1}\right)$ and such that $m\left(\mathbf{R} \backslash \cap_{n=1}^{\infty} S_{f_{n}}\right)=0$.

Proof. If $\left\{f_{n}\right\}_{n=1}^{\infty}$ is dense in $\mathscr{H}$ (or in $\Sigma_{1}$ ) then $m\left(\mathbf{R} \backslash S_{f_{n}}\right)=0$ and so $m\left(\mathbf{R} \backslash \cap_{n=1}^{\infty} S_{f_{n}}\right)=0$.

REMARKS. If $F$ is any (probability) distribution function there is a Hilbert space $\mathscr{H}$, a resolution of the identity $E(t)$, and in $\Sigma_{1}$ an $f$ such that for all $t, F(t)=F_{f}(t)$. Indeed if $\mathscr{H}=L^{2}(R, P), P$ being the measure induced on $\mathbf{R}$ by $F$, let $E(t) k$ be $\chi_{(-\infty, t]} \cdot k$ for $k$ in $\mathscr{H}$. Then the function $f$ identically 1 is in $\mathscr{H}$ and $F=F_{f}$.

More generally, if $F_{a_{1} a_{2} \cdots a_{n}}$ is a family of multidimensional (probability) distribution functions satisfying the classical Kolmogorov consistency conditions (all $a_{i}$ are members of a fixed parameter set $A$ ) and if $S=\mathbf{R}^{A}$, endowed via Kolmogorov's construction with the relevant measure $P$, then for each $a$ in $A$ and each $f$ in $L^{2}(S, P)$ let $E_{a}(t) f$ be defined by: if $\left\{r_{b}\right\}$ is in $S$ then

$$
E_{a}(t) f\left(\left\{r_{b}\right\}\right)=f\left(\left\{r_{b}\right\}\right) \text { if } r_{a} \leq t=0, \quad \text { otherwise. }
$$

Then if $f \equiv 1$ on $S$

$$
F_{a_{1} a_{2} \cdots a_{n}}\left(t_{1}, t_{2}, \ldots, t_{n}\right)=\left(\prod_{i=1}^{n} E_{a_{t}}\left(t_{t}\right) f, f\right) .
$$

Thus every stochastic process may be realized as a quantum mechanical process. By varying $f$ over $\Sigma_{1}$ in $L^{2}(S, P)$ one achieves a family of (related) stochastic processes.

If $X_{1}$ and $X_{2}$ are random variables with distribution functions $F_{1}$ and $F_{2}$, then when $X_{1}$ and $X_{2}$ are independent, the distribution function for 
$X_{1}+X_{2}$ is $F_{1} * F_{2}$, i.e.,

$$
P\left(X_{1}+X_{2} \leq x\right)=F_{1} * F_{2}(x)=\int_{\mathbf{R}} F_{1}(x-y) d F_{2}(y) .
$$

If $X_{1}$ and $X_{2}$ are not independent the formula above may fail to hold. On the other hand, if $X_{1}, X_{2}$ is realized in the manner given above, then, e.g.,

$$
\begin{aligned}
& F_{1}(x)=\left(E_{1}(x) f, f\right) \quad(f \equiv 1) \\
& F_{2}(x)=\left(E_{2}(x) f, f\right) .
\end{aligned}
$$

Now, however, even if $X_{1}$ and $X_{2}$ are not independent,

$$
P\left(X_{1}+X_{2} \leq x\right)=\int_{\mathbf{R}}\left(\left(E_{1}(x-y) d E_{2}(y)\right) f, f\right),
$$

i.e., a convolution-like formula may be used. Furthermore if $U_{a}(s)=$ $\int_{\mathbf{R}} e^{i s t} d E_{a}(t)$ then the joint characteristic function for $F_{a_{1} a_{2} \cdots a_{n}}$ is $\left(\prod_{i=1} U_{a_{i}}\left(s_{i}\right) f, f\right)$, i.e., a product-like formula even if there is no independence indicated.

\section{REFERENCES}

[1] G. Darmois, Analyse générale des liaisons stochastiques, Rev. Inst. Intern. Stat., 21 (1953), 2-8.

[2] B. R. Gelbaum, Independence of events and of random variables, Zeitschrift für Wahrscheinlichkeitstheorie, 36 (1976), 333-342.

[3] A. M. Kagan, Yu. V. Linnik and C. R. Rao, Characterization Problems in Mathematical Statistics, John Wiley \& Sons, New York, 1973.

[4] R. Hemasinha, Ph.D. dissertation, SUNY/Buffalo, 1983.

[5] Yu. V. Linnik, Decomposition of Probability Distributions, Oliver and Boyd, Ltd. Edinburgh, 1964.

[6] E. Nelson, Probability Theory and Euclidean Field Theory, in Constructive quantum field theory, Springer-Verlag, 1973.

[7] I. Segal, Tensor algebras over Hilbert spaces, I, Trans. Amer. Math. Soc., 81 (1956), 106-134.

[8] V. P. Skitovich, On a property of the normal distribution, DAN SSSR, 89 (1953), 217-217.

[9] Linear forms in independent random variables and the normal distribution law, Izvestia AN SSSR, Ser. Matem.

[10] J. von Neumann, Grundlagen der Quantenmechanik, Dover Publications, New York, 1943.

Received April 18, 1984.

SUNY / BUFFALO

BUFFALO, NY 14214-3093 
